\title{
PUBLICATIONS OF THE ASTRONOMICAL SOCIETY OF THE PACIFIC
}

\begin{tabular}{lll}
\hline \hline Vol. 101 & December 1989 & No. 646 \\
\hline \hline
\end{tabular}

\section{SPECTROSCOPY OF QUASAR CANDIDATES FROM THE CASE LOW-DISPERSION SURVEY}

\author{
D. J. THOMPSON, S. DJORGOVSKI,* AND W. N. WEIR \\ Palomar Observatory, California Institute of Technology, Pasadena, California 91125 \\ Received 1989 August 4, revised 1989 August 30
}

\begin{abstract}
We present long-slit CCD spectra of 19 stellar objects selected from the Case Low-Dispersion Northern Sky Survey. Thirteen of them are quasars, ranging in redshift from 0.0786 to 2.67 , with a median redshift of 2. CSO 203 is a broad absorption-line quasar, and CSO 38 may have substantial associated absorption in the cores of emission lines. Several other objects show some intervening absorption, and all of them are bright enough to make the follow-up studies practical. CSO 251 is a bright $\left(\sim 15^{\mathrm{m}}\right)$, previously uncataloged quasar. The remaining objects are Galactic stars, five subdwarfs, and one hot white dwarf (CSO 160).
\end{abstract}

Key words: galaxies: redshifts-quasars-redshifts-spectroscopy-stars: subdwarf

\section{Introduction}

The Case Low-Dispersion Northern Sky Survey is a slitless spectroscopy survey for blue and emission-line objects, with the limiting magnitude $B \sim 18$ (Pesch and Sanduleak 1983, 1986, 1988, 1989, and references therein; Sanduleak and Pesch 1984). The survey has produced many interesting objects: quasars, active and starforming galaxies, rare types of stars, etc. Stellar objects selected in this survey are usually designated as CSO, a convention which we follow. The information obtained from the survey plates is rather limited, and redshifts are seldom determined for quasars; follow-up slit spectroscopy is necessary in most cases.

The principal advantage of the Case and similar surveys in selecting quasars is that the objects are relatively bright. That makes them particularly useful for absorption-line studies, if the redshifts are sufficiently large. Bright, high-redshift quasars are also good candidates for gravitational lenses, as witnessed by the success of surveys conducted by Surdej and collaborators (Surdej et al. 1989, and references therein; Djorgovski and Meylan 1989). Yet, the Case survey seems to be somewhat neglected: The Hewitt and Burbidge (1987) catalog lists only $15 \mathrm{CSO}$ objects as confirmed quasars.

We have undertaken a follow-up slit spectroscopy pro-

*Alfred P. Sloan Foundation fellow. gram of bright CSO quasar candidates at Palomar Observatory, as a marginal weather backup project. Our goal is to measure the redshifts and identify objects worth pursuing in more detail.

\section{Data and Results}

The observations reported here were done on UT 1988 January 10 and 11 and UT 1988 March 10 and 11, using the Double Spectrograph (Oke and Gunn 1982) mounted in the Cassegrain focus on the Hale 200-inch (5-m) telescope. The conditions were nonphotometric, with variable transparency, and the seeing was never better than about 2 or 3 arc sec (FWHM). The slit width used was 2 arc sec and the effective spectral resolution (FWHM) was about $10 \AA$. The wavelength range covered was different for different nights, but typically was $3700 \AA-7400 \AA$, or $3600 \AA-9000 \AA$; on the night of UT 1988 January 10 there was a gap of about $500 \AA$ in the coverage between the blue and the red sides of the spectrograph. The exposure times were in the range of 900-2000 sec.

Exposures of arc lamps were used to obtain the dispersion solutions, giving the wavelength calibration rms of $\sim$ $1 \AA$. Dome flat fields and exposures of standard stars from Oke and Gunn (1983) were used to correct for the instrument response and to provide a crude flux calibration, and we estimate the zero-point uncertainties to be of the order of 0.5 . The data were reduced using standard procedures. 
A total of 19 CSO objects were observed, 13 of which one white dwarf (CSO 160). An emission-line foreground turned out to be quasars, and six Galactic stars, including galaxy was found near CSO 259. Figures 1-4 show the
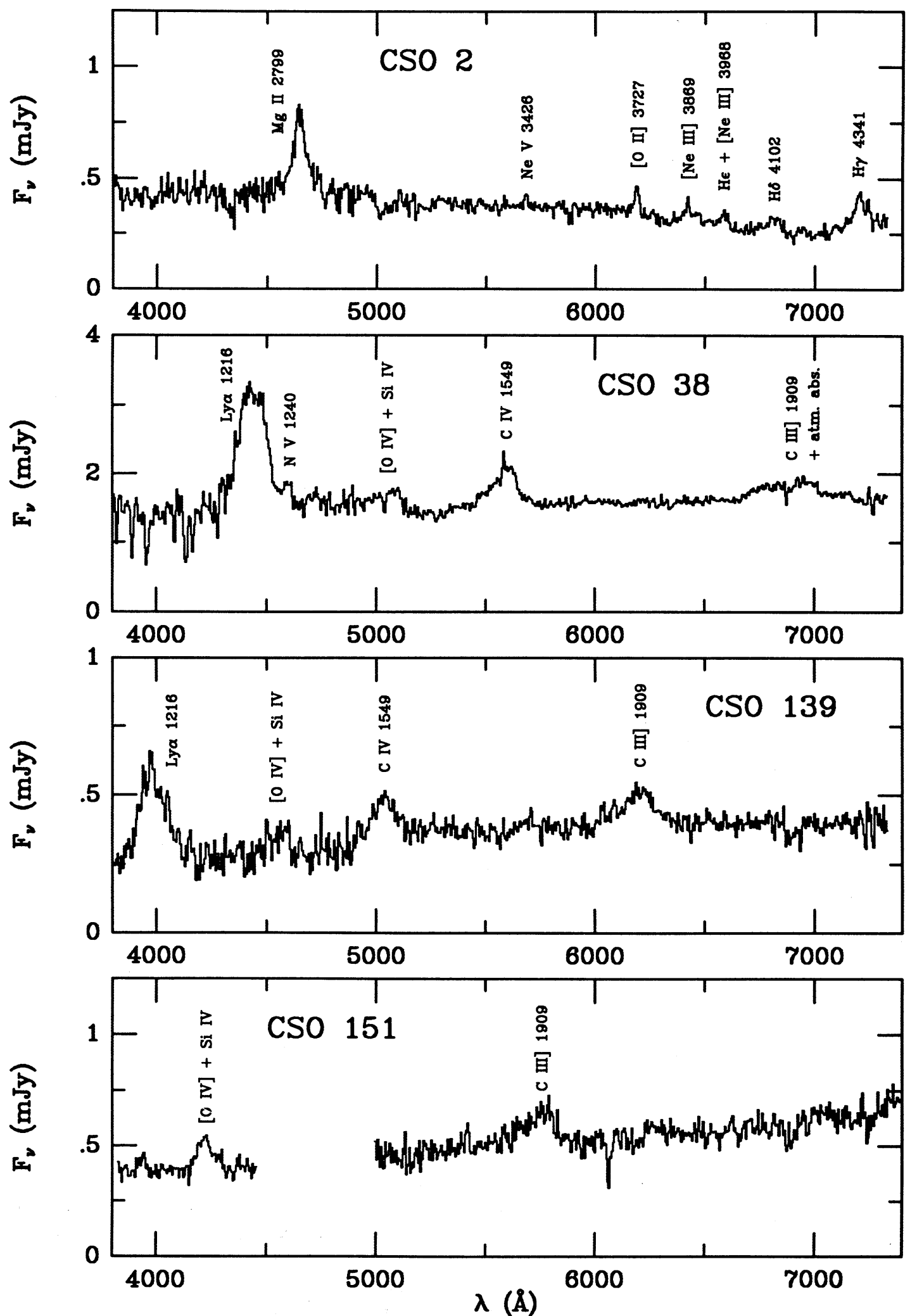

FIG. 1-Spectra of CSO 2, CSO 38, CSO 139, and CSO 151, with emission lines identified. The flux calibration zero point is uncertain by as much as $0.5 \mathrm{mag}$ and different for different objects. See the text for further details. 
SPECTROSCOPY OF QUASAR CANDIDATES

spectra of the AGNs spectroscopically identified here with the features labeled.
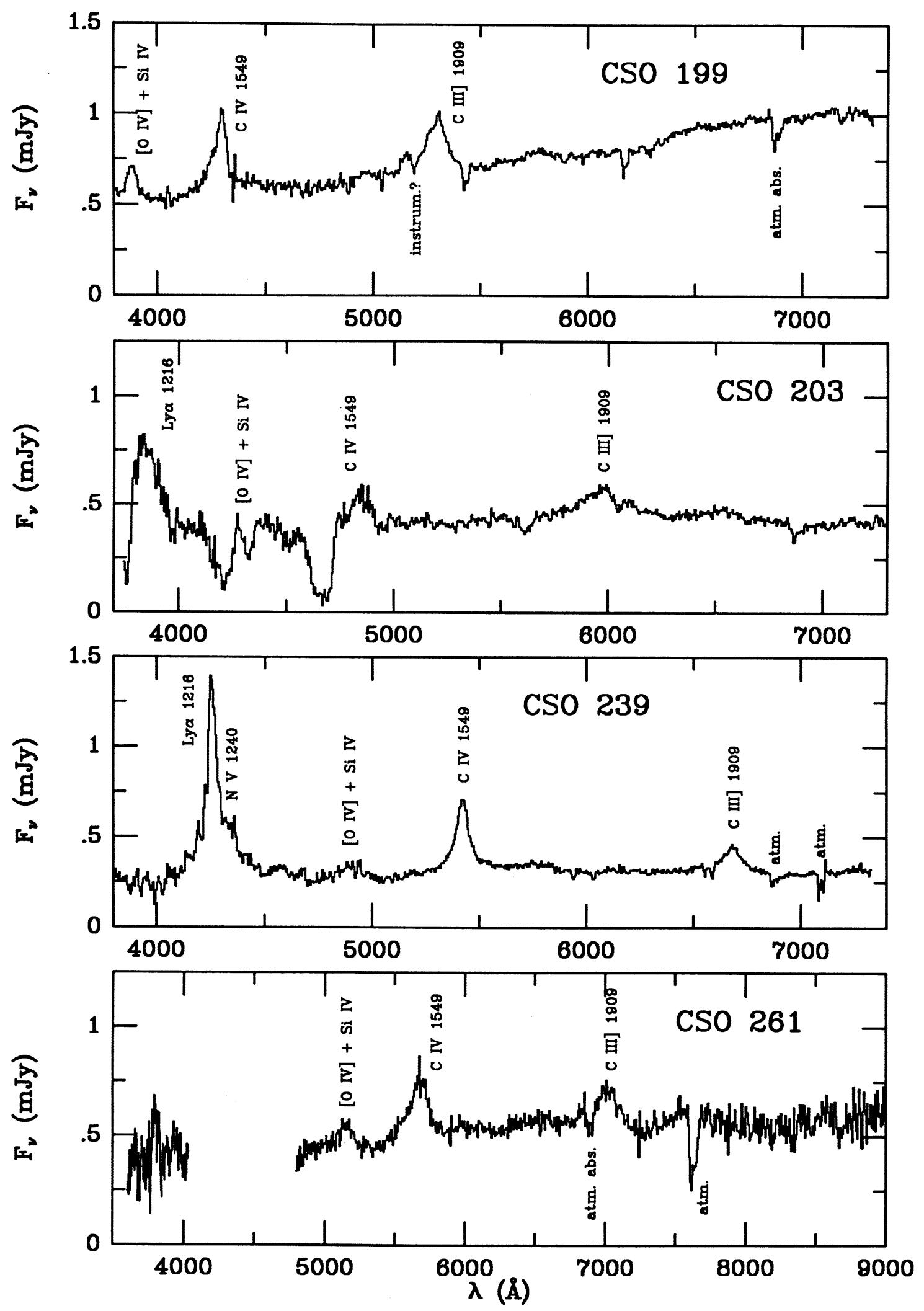

Fig. 2-As in Figure 1, but for CSO 199, CSO 203, CSO 239, and CSO 261. 

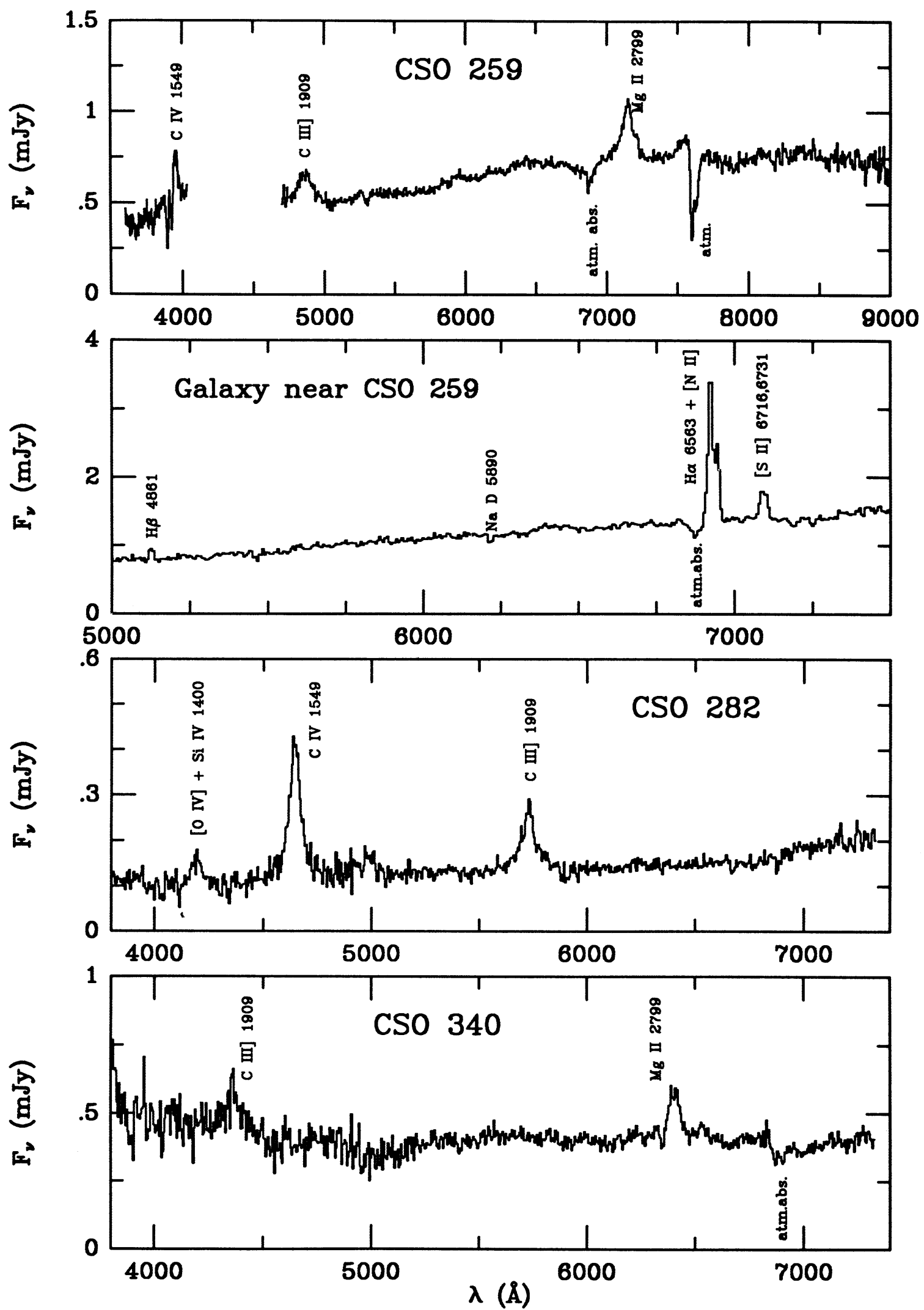

FIG. 3-As in Figure 1, but for CSO 259, foreground galaxy nearby, CSO 282, and CSO 340. 


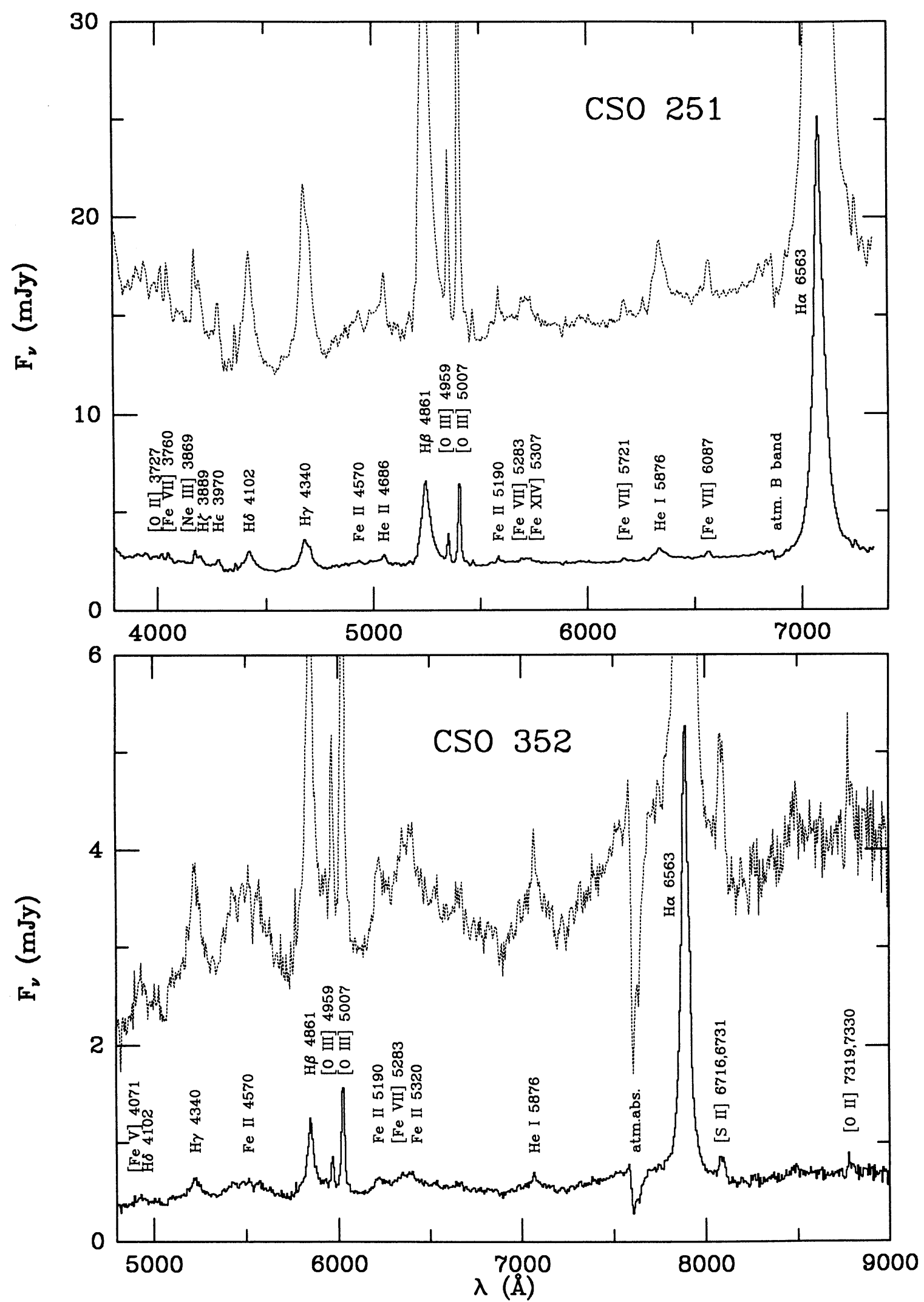

FIG. 4-Spectra of CSO 251 (top) and CSO 352 (bottom), with features labeled. Solid lines correspond to the nominal flux calibration, and dotted lines are the spectra multiplied by a factor of 6 , in order to show the detail. CSO 251 is an unusually bright object, with $\sim 15^{\mathrm{m}}$. 


\section{Comments on Individual Objects}

CSO $2=0844+295$ : Also known as Ton 11. A $17^{\mathrm{m}}$ quasar or an N-galaxy at $z=0.660 \pm 0.001$. Pesch and Sanduleak (1983) noted a possible weak emission line near $4360 \AA$, which we identify as Mg II 2799.

CSO $24=0950+294:$ A $16^{\mathrm{m}}$ subdwarf star with narrow absorption lines, probably $\mathrm{sdF}$ or later.

CSO $38=1009+299:$ A $16^{\mathrm{m}}$ quasar at $z=2.62 \pm 0.01$. Pesch and Sanduleak (1983) noted a possible emission line near $4450 \AA$, which we identify as Lyman- $\alpha$. The stubby shape of the emission lines suggests a considerable associated absorption (the $\mathrm{C}$ III] line is affected by telluric absorption as well, but that is probably a minor effect). Most of the absorption features seen blueward of the $L \alpha$ are real.

CSO $139=1224+290:$ A $17^{\mathrm{m}}$ quasar at $z=2.25 \pm$ 0.01. Sanduleak and Pesch (1984) noted a possible emission line near $3960 \AA$, which we identify as $L \alpha$.

CSO $151=1231 \pm 294$ : Also known as Ton 621. A $16^{\mathrm{m}}$ quasar at $z=2.011 \pm 0.001$. Sanduleak and Pesch (1984) noted a possible weak emission line near $4640 \AA$ which is not covered by our spectra, but it corresponds to the $\mathrm{C}$ IV $\lambda 1549$ line expected from our redshift identification. Note the absorption feature at $6065 \AA$.

CSO $160=1240+326$ : A hot, $17^{\mathrm{m}}$ white dwarf, probably DB. From the red continuum slope we estimate $T_{\mathrm{BB}} \sim$ $20,000 \mathrm{~K}$.

CSO $186=0819+363:$ A $17^{\mathrm{m}}$ subdwarf star with narrow absorption lines, probably $\mathrm{sdF}$ or later.

CSO $199=0838+359:$ A $16^{\mathrm{m}}$ quasar at $z=1.775 \pm$ 0.002 . The feature at $5195 \AA$ is probably an artifact corresponding to the spectrograph dichroic split. The absorption features near $5225 \AA$ and $6170 \AA$ are probably real.

CSO $203=0842+345$ : Broad absorption-line quasar (BALQ) at $z=2.126 \pm 0.003,17^{\mathrm{m}}$. The broad absorption features, and the emission line near $3825 \AA$ (identified here as $L \alpha$ ), were already noted by Pesch and Sanduleak (1986).

CSO $239=0952+338:$ Also known as Ton 1125. A $17^{\mathrm{m}}$ quasar at $z=2.500 \pm 0.001$. Pesch and Sanduleak (1986) noted the emission line near $4210 \AA$, identified here as L $\alpha$. Note the weak absorption features at $5940 \AA, 6035 \AA$, $6555 \AA$, and $6590 \AA$.

CSO $241=0954+341:$ A $16^{\mathrm{m}}$ subdwarf star with narrow absorption lines, probably $\mathrm{sdF}$ or later.

CSO $251=1010+361$ : Also known as Ton 1187. A very bright $\left(\sim 15^{\mathrm{m}}\right)$ quasar at $z=0.0786 \pm 0.0002$ (from the hydrogen lines alone: $\mathbf{0 . 0 7 8 4 5}$, from the [O III] lines alone: 0.07887 ; the difference is marginally significant). The lines are very luminous, and the spectrum is quite rich (see Fig. 4). It is remarkable that this object was not (to the best of our knowledge) previously noted in any of the quasar surveys or catalogs. Pesch and Sanduleak (1986) noted the emission lines near $4620 \AA(\mathbf{H} \gamma)$ and
$5240 \AA(\mathrm{H} \beta)$. It is the only CSO object without a published finding chart, but at 15 th mag one is hardly necessary.

CSO $259=1016+359:$ A $17^{\mathrm{m}}$ quasar at $z=1.552 \pm$ 0.004 . It is not clear whether the absorption features on the blue side of the C IV $\lambda 1549$ line are real. There is a foreground galaxy $\sim 45$ arc sec away in P.A. $=207^{\circ}$, at $z=$ $0.055 \pm 0.001$, with a fairly blue continuum and a lowionization, emission-line spectrum (Fig. 3), suggestive of a starburst galaxy. There is also an IRAS point source about 3 arc min to the east from the quasar. Pesch and Sanduleak (1988) note the "proximity of a group of galaxies".

CSO $261=1016+359:$ A $17^{\mathrm{m}}$ quasar at $z=2.67 \pm$ 0.01. Pesch and Sanduleak (1988) suggested that this object is a BALQ, but we find no evidence for it.

CSO $282=1036+355 \mathrm{~A}:$ A $17^{\mathrm{m}}$ quasar at $z=1.999 \pm$ 0.002. Pesch and Sanduleak (1988) noted a possible emission line near $3660 \AA$, which according to our redshift determination must be $\mathrm{L} \alpha$.

CSO $283=1036+355 \mathrm{~B}:$ An $18^{\mathrm{m}}$ subdwarf star with weak narrow absorption lines, probably sdF.

CSO $340=1123+356:$ A $17^{\mathrm{m}}$ quasar at $z=1.285 \pm$ 0.004. Pesch and Sanduleak (1988) note that the object is "located in the midst of a small group of galaxies", undoubtedly foreground. The quasar may be gravitationally (micro?) lensed.

CSO $352=1131+358:$ A $17^{\mathrm{m}}$ quasar at $z=0.2035 \pm$ 0.0005 . The iron blends are prominent in the spectrum (Fig. 4). Pesch and Sanduleak (1988) note that the object is "about 5 arc sec south of a faint galaxy"; we were not successful in obtaining its spectrum. Given the relatively low redshift of the quasar, the two may be physically associated.

CSO $362=1141+362:$ A moderately hot, $17^{\mathrm{m}}$ subdwarf star, sdA or sdF. The Balmer series is very prominent.

\section{Concluding Remarks}

Our impression is that the CSO lists provide a good yield of quasars compared to some other, similar surveys (e.g., Kiso, or even UM). However, our source selection was biased toward objects for which the original papers indicated a (possible) presence of emission lines.

Probably the most interesting objects found here are CSO 251 (because of its brightness) and CSO 38 and CSO 203 (because of the absorption features). Several other objects noted above show absorption lines, possibly Mg II $\lambda 2799$ from intervening galaxies, and may be worth following up with a higher spectral resolution.

We are indebted to the staff of Palomar Observatory for their expert help and in particular to Juan Carrasco, Dave Tennant, John Henning, and Skip Staples. We also thank Helen Johnston, Alain Picard, and Georges Meylan for their assistance with the observations and Charles Steidel 
for useful comments on the manuscript. This work was supported in part by a Caltech Institute Institute Fellowship (D.J.T.), the Alfred P. Sloan Foundation and California Institute of Technology (S.D.), and an NSF Fellow$\operatorname{ship}$ (W.N.W.).

\section{REFERENCES}

Djorgovski, S., and Meylan, G. 1989, in Gravitational Lenses, Lecture Notes in Physics, 330, ed. J. Moran et al. (Berlin: Springer Verlag), p. 173.

Hewitt, A., and Burbidge, G. 1987, Ap. J. Suppl., 63, 1.

Oke, J. B., and Gunn, J. E. 1982, Pub. A.S.P., 94, 586. . 1983, Ap. J., 266, 713.

Pesch, P., and Sanduleak, N. 1983, Ap. J. Suppl., 51, 171. . 1986, Ap. J. Suppl., 60, 543. 1988, Ap. J. Suppl., 66, 297. 1989, Ap. J. Suppl., 70, 173.

Sanduleak, N., and Pesch, P. 1984, Ap. J. Suppl., 55, 517. Surdej, J. et al. 1989, ESO Messenger, No. 55, 8. 